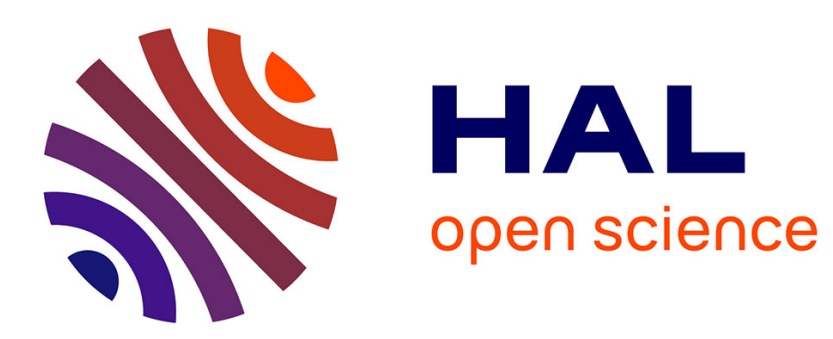

\title{
RECHERCHES SUR LA CASÉINE. I. LA CASÉINE N'EST PAS UN CORPS HOMOGĖNE. ÉTUDE DE SON FRACTIONNEMENT PAR LE CHLORURE D'AMMONIUM
}

Emile Cherbuliez, Margarethe Lily Schneider.

\section{To cite this version:}

Emile Cherbuliez, Margarethe Lily Schneider.. RECHERCHES SUR LA CASÉINE. I. LA CASÉINE N'EST PAS UN CORPS HOMOGĖNE. ÉTUDE DE SON FRACTIONNEMENT PAR LE CHLORURE D'AMMONIUM. Le Lait, 1933, 13 (123), pp.264-277. hal-00895108

\section{HAL Id: hal-00895108 https://hal.science/hal-00895108}

Submitted on 1 Jan 1933

HAL is a multi-disciplinary open access archive for the deposit and dissemination of scientific research documents, whether they are published or not. The documents may come from teaching and research institutions in France or abroad, or from public or private research centers.
L'archive ouverte pluridisciplinaire HAL, est destinée au dépôt et à la diffusion de documents scientifiques de niveau recherche, publiés ou non, émanant des établissements d'enseignement et de recherche français ou étrangers, des laboratoires publics ou privés. 
Faute de pâtures, le pays très crayeux et très sec étant entièrement livré à la culture, les vaches vivent en étable toute l'année, et sont nourries de résidus d'industrie : drèches, mélasses, et de fourrage artificiel; du moins à l'époque où nous avons analysé leur beurre (Septembre 1930). Nous n'avons pas trouvé d'indices aussi faibles dans toute la littérature beurrière :

Indice de saponification moyen : 208 ;

Indice d'acides volatils solubles moyen : 16 .

(A suivre.)

RECHERCHES SUR LA CASÉINE.

\title{
1. LA CASÉINE N'EST PAS UN CORPS HOMOGÈNE. ÉTUDE DE SON FRACTIONNEMENT PAR LE CHLORURE D'AMIVONIUM (1)
}

\author{
par \\ Emile CHeRBULIEZ et Margarethe Lity SCHNEIDER. \\ (Travail du Laboratoire de Ohimie organique de l'Université, Genève.)
}

\section{INTRODUCTION.}

La caséine d'une espèce animale donnée est une substance qui présente des propriétés remarquablement constantes lorsque, dans sa préparation à partir du lait, on a soin d'éviter tout ce qui, selon les vues actuelles, pourrait l'altérer. Cette constance des propriétés de la caséine, notamment de celle qui est retirée du lait de vache, a fait affirmer déjà à HAMMARSTEN (2), dès 1877, son homogénéité.

A priori, la constance des propriétés d'un corps tel que la caséine (3) ne prouve pas grand'chose en ce qui concerne son homogénéité, car cette constance ne s'observe que pour des échantillons obtenus par un procédé toujours le même dans ses grands traits : précipitation à un $p H$ donné, éventuellement répétition de cette opération à partir d'une solution dans de l'alcali dilué, obtenue en ayant soin de ne jamais dépasser le $p H$ 7. Les conditions de précipitation étant ainsi constantes, il n'y a aucune raison d'espérer pouvoir réaliser une séparation de colloïdes s'entraînant réciproquement lors de leur floculation dans des conditions toujours les mêmes. Cette notion de l'impossibilité de séparer les constituants éventuels de la caséine par simple répétition de la précipitation a été développée particulièrement nettement par LINDERSTRöMLANG (4).

(1) Reproduit de Helvetica Chimica Acta, 15, 597 (1932), avec l'autorisation de la Rédaction.

(2) Acta Reg. Soc. Scient. Upsal. No X (1877), cité d'après LinderströM-LANG, C. r. trav. Lab. Carlsberg 17, No 9 (1929).

(3) Dans la suite de cette note, nous entendrons par « caséine » tout court toujours la casóine du lait de vache.

(4) C. r. trav. Lab, Carlsberg 17, No 9, 2 (1929). 
Malgré la constance de ses propriétés, la caséine a été considérée par un certain nombre d'auteurs comme un mélange d'au moins deux corps différents; il y a en effet toute une série d'observations qui sont difficiles à concilier avec la notion d'homogénéité de ce corps. Parmi ces faits, citons notamment les observations suivantes.

En 1880, déjà, Danilewsky et Radenhausen (1) ont observé que la caséine est partiellement soluble dans l'alcool à $50 \%$ à l'ébullition. Cette observation, qui n'a guère été retenue, est à rapprocher peut-être d'une constatation faite presque 40 ans plus tard par OSBORNE et WAKEMAN (2). Ces auteurs ont pu retirer de la caséine, par action de l'alcool chaud, une petite quantité $(0,5 \%)$ d'un protide soluble dans l'alcool et exempt de phosphore.

En 1898, Alexander (3) a constaté que la précipitation de la easéine par le sulfate d'ammonium de la solution neutre dans de la soude caustique diluée, se fait dans un intervalle de concentrations relativement étendu, comparé au rapprochement de la limite des concentrations nécessaire pour le début d'une précipitation et suffisante pour une précipitation intégrale, pour d'autres protides.

KовRAK (4), en 1900, a fait une observation intéressante qui semble avoir passé à peu près inaperçue : il a constaté que la caséine du lait de vache, aussi bien que celle du lait de femme, est partiellement soluble dans les solutions diluées de quelques sels minéraux, tels que le chlorure de sodium, le sulfate de magnésium et le sulfate de sodium.

Tout récemment, Linderström-Lang a repris l'étude de la caséine au laboratoire de Carlsberg, avec toute la préeision et la méticulosité de cette école remarquable. Cet auteur a constaté d'abord (5) que la solubilité de la caséine dans l'acide chlorhydrique dilué en présence de chlorure de sodium, à température et concentration égales, était fonction de la quantité de caséine mise en œuvre - comme si la caséine était composée d'au moins deux fractions de solubilités différentes. Il a constaté en outre (6) qu'on pouvait obtenir des fractions qui se distinguent par leurs propriétés chimiques (composition centésimale, proportions des amino-acides dans les produits d'hydrolyse) et physico-chimiques (vitesse de coagulation par la présure, pouvoir neutralisant vis-à-vis de bases) ; il suffit de traiter la caséine par exemple par de l'alcool chlorhydrique très dilué, à $70^{\circ}$, et de précipiter les solutions obtenues, par

(1) Forsehungen auf dem Gebiet der Viehhaltung 2, 1 (1880), eité d'après $\mathrm{O}$. HaMMARsten, Z. physiol. Ch. 7, 227 (1883).

(2) J. biol, Chem. 33, 7, 243 (1918).

(3) Z. physiol. Ch. 25, 411 (1898).

(4) Plüger's Arch. ges. Physiol. 80, 69 (1900).

(5) C. $r$. trav. Lab. Carlsberg 16, No 1 (1925).

(6) Z. physiol. Ch. 176, 76 (1928); C. r. trav. Lab. Carlsberg 17, No 9 (1929). 
addition fractionnée de soude, pour obtenir toute une série de fractions légèrement mais nettement différentes les unes des autres. LINDERSTRÖM-LANG oroit pouvoir écarter l'hypothèse d'un début de désagrégation de la caséine par le traitement à l'alcool chlorhydrique en se basant sur des constatations expérimentales très nettes : non seulement l'azote formol n'est pas augmenté par ce traitement, mais encore toutes les fractions obtenues, réunies, donnent un produit qui possède toutes les propriétés physicochimiques et chimiques de la caséine primitive. Les travaux cités tendent donc à faire admettre que ce qu'on appelle caséine est un mélange d'au moins deux corps différents; mais la séparation de ces corps encore plus ou moins hypothétiques n'a pas encore pu être réalisée. LINDERSTRÖM-LANG estime que ses différentes fractions ne représentent toujours que des mélanges dans lesquels les proportions primitives des constituants sont seulement légèrement changées.

Les déterminations toutes récentes de poids moléculaire faites par SVEDBERG et ses eollaborateurs (1) par ultra-centrifugation confirment la théorie de la non-homogénéité de la caséine. La caséine "pure" est un mélange de molécules de grandeurs très variables (75.000 à 375.000$)$, tandis qu'une certaine fraction, obtenue selon LINDERSTRÖM-LANG par fractionnement dans l'alcool chlorhydrique, a présenté des molécules de grandeur constante (375.000).

Le problème de la non-homogénéité de la caséine étant ainsi posé, il faut envisager non seulement la question du nombre et de la nature des constituants ; il y a encore celle de la constance ou de la variation de leurs proportions respectives dans la "caséine " d'une espèce déterminée. Là encore, nous avons des données qui tendraient à faire admettre non seulement que la " caséine " pure est un mélange, mais encore que ce mélange, même chez un individu déterminé, n'est pas de composition constante.

H. Meier (2) a déterminé les limites inférieure et supérieure du $p$ H entre lesquelles la caséine est précipitée de la solution aqueuse de son sel alcalin, ceci en fonction de la température. $\mathrm{Il}$ constate que les limites de $p H$ entre lesquelles se fait cette précipitation sont d'autant plus rapprochées que la température est plus basse. On peut déterminer une température-limite de floculation, minimum nécessaire, même au $p H$ optimum, pour obtenir la floculation. Or cette température-limite varie non seulement pour les caséines des diverses espèces animales, mais encore, dans la même espèce, d'un individu à l'autre (notamment chez la femme). Ceci n'est pas un phénomène dû à des variations dans la composition du lait; ces différences se retrouvent encore avec les caséines isolées selon la technique

(1) Svedberg, L. M. Carpenter, B. C. Carpenter, Am. Chem. Soc. 52, 241 (1930).

(2) H. MeIER, Bioch. Z. 178, 82 (1924). 
habituelle de précipitation lente en solution diluée, à un $p \mathrm{H}$ déterminé. Avec les solutions des caséines "pures», les différences ne sont toutefois bien marquées qu'en présence de petites quantités de sels neutres, dont l'influence très nette est intéressante à noter.

\section{FRACTIONNEMENT DE LA CASÉINE PAR LES SELS NEUTRES.}

Le point de départ de nos expériences a été la constatation faite par КовRАк (1) qu'une partie de la caséine se dissout dans les solutions salines neutres, et qu'après quelques extractions répétées la caséine ne cède plus rien à la solution saline. Nous avons employé pour toutes nos expériences la "caséine selon HAMMARSTEN » telle qu'elle est fournie par la maison Schering-KaHLBaum.

Nous avons déterminé tout d'abord approximativement comment se comportait la caséine à l'égard de solutions de sulfate de magnésium de concentrations variables, en agitant des prises de $1 \mathrm{gr}$. de caséine avec $50 \mathrm{~cm}^{3}$ de la solution à étudier. Une appréciation suffisamment précise de la quantité de protide qui a passé en solution peut être basée sur la précipitation d'une quantité donnée $\left(5 \mathrm{em}^{3}\right.$ ) de la solution filtrée, limpide, par de l'acide phosphotungs. tique en solution chlorhydrique (qui ne précipite pas par le sulfate de magnésium).

Voici le résumé de nos observations :

$1 \mathrm{gr}$. caséine, $50 \mathrm{~cm}^{3} \mathrm{SO}^{4} \mathrm{Mg}+7 \mathrm{H}^{2} \mathrm{O}, 30 \%$ et plus cone.

pas de précipité

\begin{tabular}{|c|c|c|c|c|c|}
\hline & " & " & ") & " & $20 \%$ \\
\hline & n & " & » & ” & $10 \%$ \\
\hline & " & D & " & ” & $5 \%$ \\
\hline & $n$ & ” & ” & " & $3 \%$ \\
\hline & " & " & " & " & $1 \%$ \\
\hline & n & ” & ” & » & $0 \%$ (eau distillée) \\
\hline
\end{tabular}
trouble précipité abondant précipité très abond. précipité très abond. précipité faible pas de précipité

Par une comparaison systématique de quelques sels, nous arrivons au classement suivant par ordre de grandeur décroissant de la quantité de substance extraite :

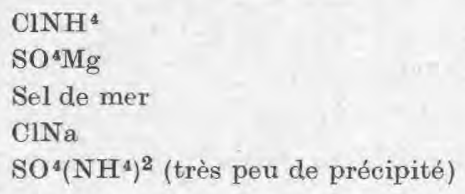

Comme l'acide phosphotungstique précipite les sels ammoniacaux, nous l'avons remplacé, dans ces essais comparatifs, par une solution d'acide tannique à $5 \%$ dans de l'acide chlorhydrique à $1,25 \%$.

Le chlorure d'ammonium se prêtant le mieux à l'étude de cette

(1) Loc. cit. 
propriété si frappante de la caséine, c'est exclusivement avec ce sel que nous avons travaillé dans la suitè.

Pour déterminer à quelle concentration le chlorure d'ammonium présente un pouvoir dissolvant maximum, nous avons dosé le protide dissous dans des extraits préparés avec des solutions de sel de concentrations variables; à cet effet, des prises de $10 \mathrm{~cm}^{3}$ des extraits ( $1 \mathrm{gr}$. de caséine séchée à l'air ( $11 \%$ d'humidité), $50 \mathrm{~cm}^{3}$ de solution saline, agités pendant 3 heures) ont été précipitées par 10 gouttes de la solution indiquée d'acide tannique (ce qui représente un faible excès du réactif tannique); les précipités ont été filtrés, lavés jusqu'à disparition $\mathrm{du} \mathrm{Cl}^{\prime}$ avec de l'eau distillée et séchés à poids constant à $50-60^{\circ}$. Comme le montre la figure 1, l'extraction est maximum pour une concentration de $5 \%$. A cette concentration, le poids du précipité tannique représente $22 \%$ du poids de la caséine. Comme ces précipités tanniques contiennent environ $8,3 \% \mathrm{~N}$, cela correspond à $13 \%$ de l'azote total de la caséine mise en œuvre.

Nous avons examiné aussi jusqu'à quel point la quantité de produit extrait dépendait de la proportion entre la caséine et le volume de la solution saline. Le total du poids du précipité tannique

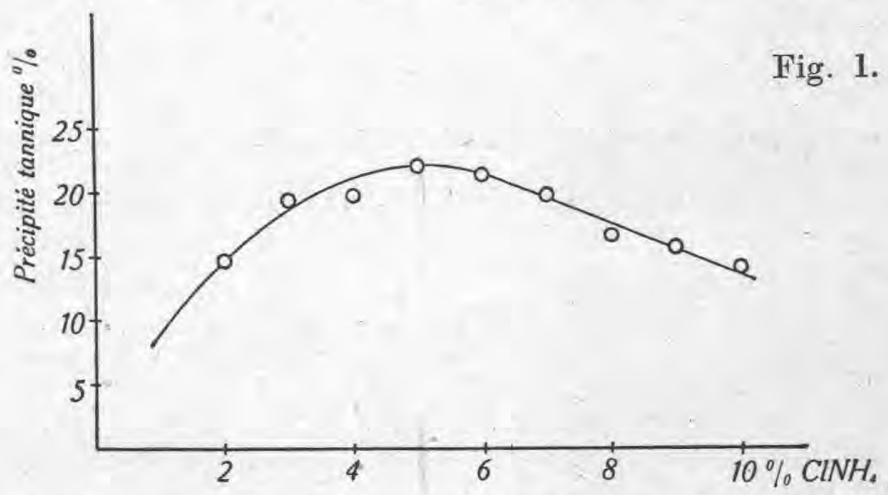

passe de $22 \%$ dans l'extrait préparé avec 50 parties de solution à $28 \%$ (100 p. de solution), $30 \%$ (200 p. de solution), $34 \%$ (300 p. de solution) et $32 \%$ (500 p. de solution). On voit que dès $100 \mathrm{p}$. de solution, l'augmentation de la quantité extraite est très petite; à partir de $200 \mathrm{p}$. de solution, l'azote extrait représente environ $17 \%$ de l'azote de la caséine primitive. Pour la préparation de la fraction soluble, nous nous sommes contentés de faire deux extractions avec 100 parties de solution; pour éliminer aussi complètement que possible la partie soluble du résidu insoluble, nous avons fait en tout 7 extractions; à partir de la septième extraction, la quantité de substance dissoute était très faible et correspondait à peu près à la solubilité dans l'eau, indiquée pour la caséine $(0,1 \%)$. 
La précipitation par le tanin est très commode comme indication qualitative de la présence d'un protide dans une solution ou pour son dosage rapide, mais elle ne se prête naturellement pas à la séparation de ce corps de sa solution. La fraction soluble de la caséine se comporte comme une globuline. Elle peut être précipitée par dialyse, par addition de sels (chlorure de sodium, sulfate d'ammonium), ou encore par un acide (acide chlorhydrique dilué). La solution saline n'est pas précipitée par l'alcool, qui précipite le sel, mais elle est précipitée par addition de 4 volumes d'acétone (l'acétone ne provoque une cristallisation du sel dans une solution de ehlorure d'ammonium à $5 \%$, qu'à partir de l'addition d'au moins 8 volumes). Pour obtenir rapidement un produit pur et exempt autant que possible de cendres, c'est au dernier procédé que nous nous sommes adressés.

\section{FRACTIONNEMENT DE LA CASÉINE PAR LE CHLORURE D'AMIMONIUM: CASÉINE $\alpha$ ET CASÉINE $\beta$}

On sait combien il est difficile d'atteindre un état d'équilibre vrai à partir d'un corps solide, amorphe et non homogène, qui, au contact du dissolvant choisi, ne passe que partiellement en solution colloïdale. Pour séparer la caséine en deux fractions, l'une soluble dans le chlorure d'ammonium, l'autre insoluble, il semble tout indiqué de procéder comme suit : partir d'une solution de caséine dans de la soude ou de l'ammoniaque diluées contenant du chlorure d'ammonium, transformer par addition de la quantité nécessaire d'acide chlorhydrique dilué (à $5 \%$ de $\mathrm{ClNH}^{4}$ ) la base employée en chlorure, et réaliser ainsi une séparation intégrale de la fraction soluble dans le chlorure d'ammonium dilué, qui restera en solution, de l'autre fraction, qui devrait précipiter. Or, lorsqu'on procède ainsi, on constate qu'après addition de la quantité nécessaire d'acide chlorhydrique à une solution de caséinate neutre en présence de chlorure d'ammonium, il n'y a aucune précipitation; ce n'est qu'après avoir ajouté un excès d'acide chlorhydrique que la caséine dissoute précipite plus ou moins complètement, selon la quantité d'acide ajoutée. On ne peut done pas atteindre un état d'équilibre entre les deux fractions présumées de la caséine, l'une soluble, l'autre insoluble dans le chlorure d'ammonium dilué neutre, en partant d'une solution de caséinate. Ce fait n'est nullement en contradiction avec cet autre fait que la caséine solide ne passe que partiellement en solution au contact de chlorure d'ammonium dilué. Il peut s'agir de deux fraetions dont les vitesses de peptisation sont très différentes; ou bien la fraction "soluble» peut servir de colloïde protecteur à la fraction "insoluble» qui se trouve déjà en solution, sans pouvoir en provoquer la peptisation lorsque cette 
dernière fraction est floculée. Comme nous le montrerons plus loin, la réalité de l'existence d'au moins deux fractions différentes dans la caséine est mise en évidence, non seulement par leur comportement à l'égard d'une solution de chlorure d'ammonium, mais encore par de nombreuses différences dans leurs propriétés chimiques. Sans vouloir discuter dès maintenant l'interprétation des phénomènes de solubilité dont nous venons de parler, nous les signalons pour motiver notre manière de procéder dans le fractionnement par le chlorure d'ammonium.

Les résultats des études préliminaires nous ont dicté la méthode suivante pour la séparation de la easéine en deux fractions, l'une insoluble dans le chlorure d'ammonium, que nous désignerons provisoirement par caséine $\alpha$, l'autre soluble, la caséine $\beta$.

On extrait $5 \mathrm{gr}$. de caséine suspendus dans 100 parties $\left(500 \mathrm{~cm}^{3}\right)$ d'une solution du sel à $5 \%$ en secouant dans un flacon rodé, à la machine, pendant 3 heures. La solution est centrifugée un quart d'heure à 4.000 tours, la solution faiblement opalescente est décantée du dépôt dans un flacon de 21 . bouché à l'émeri (vol. : $450 \mathrm{~cm}^{3}$ ) et additionnée de 4 volumes $\left(1.800 \mathrm{~cm}^{3}\right)$ d'acétone. De la solution qui se trouble se dépose assez lentement un précipité volumineux et incolore de caséine $\beta$. Après quelques heures de repos, on filtre le précipité.à la trompe sur du coton serré (débarrassé de son apprêt par un traitement à la soude bouillante très diluée et par un lavage soigneux

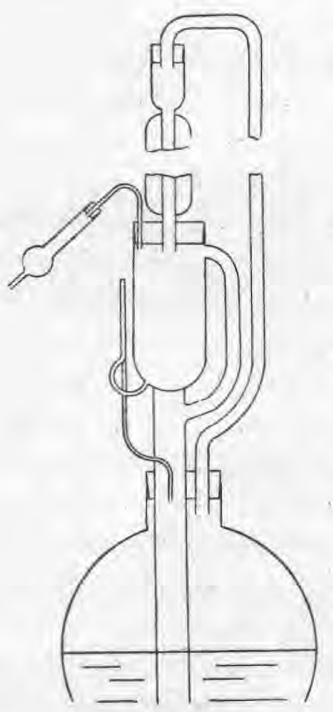

Fig. 2.

à l'eau). Comme la centrifugation ne fournit pas une solution tout à fait limpide, nous avons redissous la caséine $\beta$ dans du chlorure d'ammonium, pour la reprécipiter. On introduit à eet effet le coton avec le précipité dans un flacon rodé avec $400 \mathrm{~cm}^{3}$ de solution, on secoue pendant une heure, on centrifuge la solution ópalescente dans laquelle le précipité s'est entièrement dissous, on décante du faible dépôt formé et on filtre le liquide obtenu. Cette solution presque limpide (390 $\mathrm{cm}^{3}$ ) est précipitée de nouveau par 4 volumes d'acétone. Le précipité est filtré sur coton, lavé à l'acétone $(80 \%)$, en ayant soin de garder le précipité toujours couvert de liquide ; on introduit le précipité plié dans le coton dans un appareil Soxhlet, modifié de manière à réaliser une extraction à la température ordinaire (v, fig. 2). On extrait le précipité d'abord à l'acétone (1 h.), puis à l'éther (1 h. ), on l'introduit rapidement dans un dessiccateur à vide et on le conserve 
sur du chlorure de calcium. On obtient ainsi la easéine $\beta$ sous forme d'une poudre blanche, très volumineuse et très hygroscopique.

Nous insistons sur le traitement du précipité tel qu'il est décrit ; il est absolument indispensable que la fraction soluble, très hygroscopique, soit débarrassée aussi complètement que possible de l'eau, et ceci par extraction avec l'acétone et l'éther, avant d'être séchée dans le vide, si on veut obtenir un produit pulvérulent. La dessiccation en présence d'eau conduit invariablement à un produit complètement racorni, qui ne se laisse pas détacher du filtre et qui n'est guère pulvérisable.

Pour obtenir un produit pur, il faut avoir soin d'employer des réactifs très purs (chlorure d'ammonium et acétone).

Lors de nos premiers essais, la teneur en soufre des deux fractions était si élevée (par ex. 1,38\% pour « et $2,35 \%$ pour $\beta$ ), qu’elle correspondait à une quantité totale de soufre bien supérieure au soufre primitif (caséine : $\mathrm{S}=0,64 \%$ ). Cette anomalie est évidemment due au fait que les fractions, notamment la fraction $\beta$, fixent les traces d'ion sulfurique que peuvent contenir les solutions. A ce point de vue, il faut vérifier la pureté non seulement du sel, mais aussi de l'acétone; un produit commercial d'acétone puriss. donnait par exemple un trouble visible lorsqu'on ajoutait à $10 \mathrm{~cm}^{3}$ un volume égal d'eau distillée et quelques gouttes de solution de chlorure de baryum. Pour purifier l'acétone, il faut distiller ce dissolvant en évitant les bouchons en caoutchouc (le contact des vapeurs avec le caoutchoue introduit des traces de soufre qui finissent évidemment par s'oxyder en ion sulfurique). Le chlorure d'ammonium peut être purifié par recristallisation en présence de chlorure de baryum, suivie d'une sublimation. Ainsi préparés, les réactifs se sont montrés exempts de $\mathrm{SO}^{\text {s.t }}$ (par le chlorure de baryum), Ca* (par l'oxalate), PO ${ }^{\text {dIII }}$ (par le molybdate d'ammonium).

Pour préparer la caséine $\alpha$, on suspend le résidu insoluble de la première extraction dans une nouvelle portion de 100 p. $\left(500 \mathrm{~cm}^{3}\right)$ de solution, on agite 1/2 heure, on centrifuge, etc. En prélevant après chaque centrifugation $2 \mathrm{~cm}^{3} \mathrm{du}$ liquide, et en les additionnant de $8 \mathrm{~cm}^{3}$ d'acétone, on obtient une gamme de précipités de volume rapidement décroissant ; nous avons indiqué déjà plus haut que, dès la septième extraction, la quantité de produit qui passe en solution est constante et excessivement faible. La caséine traitée 7 fois par la solution saline est suspendue dans $500 \mathrm{~cm}^{3}$ d'eau distillée en présence d'un peu de toluène; on laisse reposer quelques heures à la glacière, on filtre et lave à l'eau distillée jusqu'à disparition de l'ion chlore. Le résidu humide est traité ensuite au Soxhlet modifié, d'abord à l'acétone, puis à l'éther, et séché dans le vide.

Le rendement en caséine $\beta$ a été en moyenne de $11 \%$; la caséine 
$\alpha$ n'est obtenue qu'avec des pertes assez considérables dues à l'entraînement mécanique d'une certaine quantité de produit dans les nombreuses opérations de décantation et de transvasage; nous n'en avons obtenu que $50 \%$ de la caséine primitive. Comme d'autre part la proportion d'azote de la caséine qui passe en solution s'élève jusqu'à $17 \%$ de l'azote total (v. plus haut, p. 268), on arrive à la conclusion que le rapport de $\beta$ à $\alpha$ doit être compris entre $1: 8$ et $1: 5$.

\section{4. ÉTUDE DES FRACTIONS $\propto$ ET $\beta$.}

La première question qui se pose, est de savoir si on est en droit de considérer les deux fractions $\alpha$ et $\beta$ comme des produits dont le mélange constitue la substance qu'on appelle caséine, ou si ces fractions sont tout simplement des produits résultant d'un début de désagrégation hydrolytique du protide primitif.

A priori, une hydrolyse de la caséine dans nos conditions de travail paraît très peu probable. La caséine est extrêmement sensible à l'action de l'eau dès que le $p H$ dépasse 7, mais en milieu très faiblement acide, elle est considérée à bon droit comme parfaitement stable. Or, le $p \mathrm{H}$ de nos solutions dans le chlorure d'ammonium est d'environ 4,6 ; c'est done une très faible acidité. En outre, toutes les opérations se font à la température ordinaire.

La composition centésimale (1) des deux fractions s'écarte très peu de celle de la caséine primitive, à l'exception du phosphore et du soufre, qui présentent une variation relative très nette. Ces variations sont cependant telles que le calcul de la composition de la caséine, basé sur les analyses des deux fractions, et sur une proportion de $7 \alpha$ à $1 \beta$, conduit aux chiffres trouvés effectivement pour la caséine.

\begin{tabular}{|c|c|c|c|c|c|}
\hline & C & $\mathrm{H}$ & $\mathrm{N}$ & S & $\mathbf{P}$ \\
\hline Caséine. & 52,45 & 7,21 & 15,59 & 0,64 & 0,84 \\
\hline Caséine $\alpha \ldots \ldots \ldots$ & 52,52 & 7,12 & 15,25 & 0,61 & 0,72 \\
\hline Caséine $\beta \quad \ldots \ldots \ldots \ldots \ldots$ & 52,60 & 6,88 & 15,34 & 0,75 & 2,32 \\
\hline
\end{tabular}

L'absence de fortes variations dans la composition centésimale ne saurait peser d'un grand poids comme argument contre l'hypothèse d'une hydrolyse, lorsqu'il s'agit de substances d'un poids moléculaire très élevé. Mais dans le cas particulier de la caséine, on sait que la dégradation en milieu neutre ou alcalin débute par la mise en liberté d'acide phosphorique. C'est à ce point de vue qu'il est intéressant de noter que, si le traitement au chlorure d'ammonium conduit à la séparation d'une fraction soluble plus riche en phos-

(1) On trouvera réunies à la fin de cette note les données analytiques se rapportant à ce paragraphe. 
phore que la caséine, il ne s'accompagne d'aucune libération d'acide phosphorique ; ceci ressort non seulement des chiffres trouvés pour le phosphore dans les deux fractions, mais surtout du fait que dans les eaux-mères acétoniques, après élimination de l'acétone au bainmarie, on ne trouve pas de phosphates précipitables par le molybdate d'ammonium.

Ce qui parle nettement en faveur de l'intégrité de nos fractions, ce sont les chiffres obtenus lors du dosage, soit de l'azote formol, soit du poids équivalent à la neutralisation à la phénolphtaléine. En cas d'hydrolyse, l'azote formol devrait augmenter, le poids équivalent à la neutralisation à la phénolphtaléine devrait diminuer. Nos chiffres pour la easéine ne sont pas exactement superposables à ceux qu'on trouve indiqués; ce qui est essentiel, c'est que les chiffres obtenus dans des conditions déterminées et identiques pour la caséine et les deux fractions, sont très rapprochés les uns des autres. Ajoutons que le poūvoir rotatoire des deux fractions ne se distingue guère de celui de la caséine.

\begin{tabular}{|c|c|c|c|}
\hline 1 & $\begin{array}{c}1 \text { gr. neutr. à } \\
\text { la phénolphtaléine } \\
\mathrm{cm}^{3} \mathrm{NaOH} 0,1-n\end{array}$ & $\begin{array}{l}\text { Azote formol } \\
\text { en } \% \text { de l'azote } \\
\text { total }\end{array}$ & $\begin{array}{c}{[\alpha]_{\mathrm{D}} \text { en sol, } 1 \%} \\
\text { dans } \mathrm{NaOH} 0,01-\mathrm{n}\end{array}$ \\
\hline Caséine $\ldots \ldots \ldots \ldots \ldots$ & 10,75 & 2,1 & $-111^{\circ}$ \\
\hline Caséine $\alpha \ldots \ldots \ldots \ldots$ & 10,33 & 2,0 & $-111^{\circ}$ \\
\hline Caséine $\beta \ldots \ldots \ldots \ldots$ & 10,15 & 1,7 & -1110 \\
\hline
\end{tabular}

Nous considérons par conséquent comme établi que nos caséines * et $\beta$ ne constituent pas des produits de désagrégation hydrolytique de la caséine primitive, mais qu'elles sont contenues dans la caséine primitive, qui n'est pas un corps homogène mais un mélange.

Si les indications qui précèdent font ressortir surtout les propriétés que les deux fractions partagent avec la caséine primitive, elles ont d'autre part toute une série de propriétés qui les différencient l'une de l'autre, en rapprochant naturellement la plus abondante, $\alpha$, de la caséine entière, tandis que la caséine $\beta$ possède des caractères très divergents.

Dans leurs solubilités d'abord : la fraction $\alpha$ se comporte à peu près comme la caséine, tandis que la fraction $\beta$ se rapproche à ce point de vue des globulines. Comme les protides de ce dernier groupe, la caséine $\beta$ est insoluble dans l'eau distillée, mais soluble dans les solutions salines diluées; elle peut en être précipitée soit par dialyse, soit par salage (avec les chlorures de sodium et d'ammonium et avec les sulfates de magnésium et d'ammonium); elle est précipitée en outre de sa solution saline par acidulation au $p \mathbf{H} 3,6$ 
environ. La solution de $\beta$ dans le chlorure d'ammonium à $5 \%$ se trouble à l'ébullition ; ce trouble disparaît par refroidissement.

Les deux fractions se distinguent encore nettement dans leur comportement vis-à-vis de la pyridine à $70 \%$. $\beta$ se dissout dans 200 p. de pyridine aqueuse rapidement et intégralement à la température ordinaire; $\alpha$ ne cède presque rien à la pyridine aqueuse, même après plusieurs heures de contact. De nouveau, la caséine primitive se comporte comme un mélange : traitée avec de la pyridine à $70 \%$, elle se dissout partiellement.

Ce qui rapproch? par contre les deux fractions, c'est qu'elles ont toutes les deux des propriétés nettement acides, et possèdent pour la neutralisation à la phénolphtaléine pratiquement le même poids équivalent (celui de la caséine entière). Grâce à ce caractère acide, la caséine $\beta$ se dissout aussi facilement que * ou la caséine entière dans l'eau dès que le $p \mathrm{H}$ est supérieur à 7 .

Les limites de concentration du salage au sulfate d'ammonium. peuvent également servir à caractériser nos deux fractions. En solution neutre, dans de la soude centinormale, la caséine $\alpha$ est précipitée à partir de $22 \%$ de saturation ; $\beta$ ne précipite qu'à partir de $32 \%$ de saturation. Ces chiffres sont intéressants à rapprocher de ceux qu'AlEXANDER (1) avait déjà trouvés pour la caséine : le caséinate de sodium en solution neutre commence à précipiter nettement à partir de $22 \%$ de saturation, mais une partie appréciable ne précipite qu'à $30 \%$. Nous retrouvons approximativement ces deux limites, chacune pour une de nos frastions.

La réaction au sulfure de plomb (caractéristique des dérivés de la cystine) est très faible pour la caséine. On sait depuis les travaux de Mörner (2) que la caséine ne contient qu'une part très faible de son soufre sous forme de dérivés de la cystine. Nos deux fractions se distinguent l'une de l'autre en ce sens que $\alpha$ donne la réaction à peu près comme la caséine, tandis que $\beta$ ne la donne que beaucoup plus faiblement, malgré sa teneur plus élevée en soufre. Il sera intéressant de vérifier si $\beta$ fournit la méthionine (ce nouvel amino-acide soufré découvert dans la caséine (3)) en proportion plus élevée que la caséine.

La coagulabilité par la présure est nettement différente chez les deux fractions. En solution à $3 \%, \alpha$ est coagulé comme la cáséine, avec formation d'un coagulum assez ferme pour qu'on puisse retourner l'éprouvette sans provoquer la dislocation du caillot. $\beta$ par contre, à la même concentration, coagule mal et donne lentement un coagulum très mou, qui ne supprime aucunement la fluidité de la solution.

(1) Loe, cit.

(2) Z. physiol. Ch. 34, 295 (1901-02).

(3) J. H. Mueller, J. biol. Chem. 56, 157 (1923). 


\section{RÉSUMÉ.}

Nous croyons avoir montré que la caséine n'est pas un corps homogène, mais un mélange d'au moins deux substances différentes, séparables par un traitement au moyen d'une solution diluée de chlorure d'ammonium.

La fraction $\alpha$, insoluble dans la solution saline, possède à peu près les propriétés de la caséine dont elle constitue la majeure partie (8/9 à 4/5). La caséine $\beta$, soluble dans le chlorure d'ammonium dilué, se distingue très nettement de la fraction insoluble, non seulement par sa solubilité, mais encore notamment par sa teneur en phosphore et en soufre, et par son comportement lors du salage et de l'emprésurage.

Il va de soi que le problème de la non-homogénéité de la caséine est Join d'être résolu par ces expériences. Nous insistons en particulier sur le fait que nos résultats ne nous permettent pas de nous prononcer sur l'homogénéité éventuelle des deux fractions obtenues, homogénéité qui nous paraît peu probable.

\section{DONNÉES ANALYTIQUES.}

a) Composition centésimale (1).

Pour gagner de la place, nous n'indiquerons le détail des chiffres que pour les données les plus importantes, c'est-à-dire pour l'azote, le soufre et le phosphore. Les substances ont été séchées à poids constant dans le vide sur de l'anhydride phosphorique à $50-60^{\circ}$.

Caséine : $\quad 0,2550 \mathrm{gr}$. ont neutr. $28,40 \mathrm{~cm}^{3}$ d'acide 0,1-n.

15,730 et $12,473 \mathrm{mgr}$. ont donné 0,710 et $0,605 \mathrm{mgr}$. SO ${ }^{4} \mathrm{Ba}$.

13,917 et $16,379 \mathrm{mgr}$. ont donné 8,070 et $9,540 \mathrm{mgr}$. [PO4.12 $\mathrm{MO}^{3}$ ] $\left(\mathrm{NH}^{4}\right)^{3}$.

Trouvé N 15,$59 ; \mathrm{S} 0,62-0,66 ; \mathrm{P} 0,84-0,84 \%$.

Caséine $\alpha$ : 5,352 mgr. ont donné $0,729 \mathrm{~cm}^{3} \mathrm{~N}^{2}\left(21^{\circ}, 725 \mathrm{~m} / \mathrm{m}\right)$.

21,880 et $18,955 \mathrm{mgr}$. ont donné 0,887 et $0,905 \mathrm{mgr}$. SO4Ba.

8,186 mgr., colorimétrie d'après EMBDEN, test 0,05 mgr. P, lecture $15,5: 100$.

Trouvé N 15,25 ; S $0,56-0,65 ; \mathrm{P} 0,72 \%$.

Caséine $\beta$ : 2,018 mgr, ont donné $0,280 \mathrm{~cm}^{3} \mathrm{~N}^{2}\left(21^{\circ}, 724 \mathrm{~m} / \mathrm{m}\right)$.

$10,720 \mathrm{mgr}$. ont donné $0,583 \mathrm{mgr}$. SO $\mathrm{Ba}$.

$3,710 \mathrm{mgr}$. (EMBDEN), test 0,05 mgr. P, lecture $42: 100$.

Trouvé N 15,$25 ; \mathrm{S} 0,75 ; \mathrm{P} 2,32 \%$.

b) Equivalent à la phénolphtaléine et azote formol.

On dicsout environ $0,1 \mathrm{gr}$. de substance dans $15 \mathrm{~cm}^{3}$ d'eau distillée, bouillie et refroidie, et 5,00 $\mathrm{cm}^{3} \mathrm{NaOH}$ 0,1-n, en activant la dissolution en écrasant les gru-

(1) Les micro-analyses ont été faites pour la caséine par G. WEILER, Berlin, et pour les caséines $\alpha$ et $\beta$, par M. FURter, Zurich, que nous remercions de leur concours d'autant plus précieux que l'hygroseopicité et la difficile oxydabilité de ces corps en ont rendu l'analyse délieate. 
meaux avec une baguette. Dès que la solution est intégrale, on titre l'excès d'alcali par de l'acide ehlorhydrique décinormal (microburette), en présence de phénolphtaléine.

Le dosage de l'azote formol se fait selon la technique habituelle dans la solution neutre résultant du titrage précédent, en la ramenant à une teinte rose pâle par de la soude diluée (comparaison à une solution type), en ajoutant du formol neutre et en ramenant la solution décolorée par le formol, à la teinte rose type.

\begin{tabular}{|c|c|c|c|}
\hline & $\begin{array}{l}\text { Poids } \\
\text { gr. }\end{array}$ & $\begin{array}{c}\mathrm{cm}^{3} \mathrm{NaOH} \text { 0,1-n } \\
\text { (neutr. à la } \\
\text { phénolphtaléine) }\end{array}$ & $\begin{array}{c}\mathrm{em}^{3} \mathrm{NaOH} 0,1-\mathrm{n} \\
\text { (azote formol) }\end{array}$ \\
\hline Caséine $\ldots \ldots \ldots \ldots$ & 0,1023 & 1,10 & 0,24 \\
\hline Caséine $\alpha \ldots \ldots \ldots \ldots$ & 0,1064 & 1,10 & $\therefore \quad 0,23$ \\
\hline Caséine $\beta \ldots \ldots \ldots \ldots$ & 0,0886 & 0,90 & 0,17 \\
\hline
\end{tabular}

c) Pouvoir rotatoire.

On dissout environ $0,1 \mathrm{gr}$. de substance dans de la soude centinormale et on porte, avec cette soude, à $10 \mathrm{~cm}^{3}$. Lectures faites à $20^{\circ}, 1=1 \mathrm{dm}$.

$$
\begin{aligned}
& \text { caséine } 0,1013 \text { gr., } \alpha=-1,13^{\circ},[\alpha]_{D}^{20^{\circ}}=-111^{\circ} \\
& \text { caséine } \alpha \quad 0,1034 \text { gr., } \alpha=-1,15^{\circ},[\alpha]_{D}^{20^{\circ}}=-111^{\circ} \\
& \text { caséine } \beta \quad 0,0993 \text { gr., } \alpha=-1,10^{\circ},[\alpha]_{D}^{20^{\circ}}=-111^{\circ}
\end{aligned}
$$

d) Solubilité dans la pyridine à $70 \%$.

$0,01 \mathrm{gr}$. de produit $+2 \mathrm{~cm}^{3}$ de pyridine à $70 \%$.

Caséine : la substance se dissout en partie. Le filtrat est légèrement louche ; $1 \mathrm{~cm}^{3}$ de filtrat évaporé au bain-marie laisse un résidu appréciable qui donne une forte réaction du biuret.

Caséine \% : la substance n'a pas l'air d'être modifiée, même après plusieurs heures de repos. Le filtrat est parfaitement limpide. Le résidu d'évaporation de $1 \mathrm{~cm}^{3}$ de filtrat est minime ; repris dans une goutte de potasse $0,01-n$ et additionné d'une goutte d'une solution de sulfate de cuivre très diluée, il donne une coloration biurétique à peine visible. La sensibilité de la réaction du biuret est d'environ 1 : 1.300 ; la quantité de protide présent dans les II gouttes de liquide de la réaction du biuret (moins de $0,1 \mathrm{~cm}^{3}$ ) doit être inférieure à $0,1 \mathrm{mgr}$., de sorte que la caséine $\alpha$ a dû céder moins de $2 \%$ à la pyridine.

Caséine $\beta$ : dissolution rapide et intégrale; la solution est légèrement louche, à peu près comme le filtrat obtenu dans l'essai avec la caséine entière. Le résidu d'évaporation de $1 \mathrm{~cm}^{3}$ de solution donne naturellement une forte réaction du biuret.

e) Coagulation par la présure.

Selon les indications récentes de Porcher (1), on prépare une solution de (1) Le lait au point de vue colloïdal. Lyon, 1930. 
caséinate et de phosphate de calcium, se rapprochant dans son état micellaire, sa concentration et son $p$ H du lait (par litre : 30 gr. de caséine, 1,100 gr. d'oxyde de calcium, $0,7498 \mathrm{gr}$. d'acide phosphorique ; $p \mathrm{H}=7$ ).

A cet effet, on empâte $0,15 \mathrm{gr}$. de substance sèche avec un peu d'eau, en la broyant dans un mortier. A la pâte homogène, on ajoute goutte à goutte, en broyant toujours, $4,9 \mathrm{~cm}^{2}$ d'eau de chaux à $1,12 \mathrm{gr}$. CaO/1. Le caséinate de calcium, d'abord blanchâtre, prend un aspect de colle opalescente. Dans ce caséinate, on introduit par très petites gouttes (microburette) espacées, en agitant énergiquement, l'acide phosphorique sous forme de $0,58 \mathrm{~cm}^{3}$ d'une solution $1 / 15$-mol. De cette manière, il ne se produit aucun précipité visible, et on obtient un liquide laiteux d'apparence parfaitement homogène.

Pour l'essà à la présure, la solution est portée dans un bain-marie à $40^{\circ}$ et additionnée de I goutte d'extrait de présure commerciale.

La caséine entière fait prise immédiatement, le caillé est assez ferme pour qu'on puifse retourner l'éprouvette sans qu'il se détache. La caséine $\alpha$ se comporte comme la caséine entière. Avee la caséine $\beta$ par contre, il ne se forme que lentement un coagulum très mou; il n'est pas question de pouvoir retourner l'éprouvette.

\section{DOSAGE DES MATIËRES GRASSES DU LAIT}

ETUDE COMPARATIVE DE L'EXTRACTION PAR L'ETHER ALCOOLISÉ DANS L'APPAREIL DE SOXHLET (MÉTHODE OFFICIELLE) ET DE L'EXTRACTION PAR L'ALCOOL DANS L'APPAREIL DE KUMAGAWA ET DANS L'APPAREIL DE SOXHLET (1)

$\begin{array}{lcc}\text { J. E. LOBSTEIN } & \text { et } & \text { M. FLATTER } \\ \text { Professeur } & & \text { Assistant }\end{array}$

à la Faculté de Pharmacie de Strasbourg.

Si l'on considère les différentes méthodes qui ont été préconisées pour le dosage des matières grasses dans le lait, on est frappé par leur multitude et leur diversité, et il semble logique de penser que le grand nombre des techniques proposées prouve implicitement qu'aueune d'elles n'est parfaite. Sans prétendre les énumérer toutes, - énumération qui risquerait fort d'ailleurs de demeurer incomplète malgré tout, car chaque laboratoire a pour ainsi dire son procédé propre ou une modification particulière d'une méthode connue, nous en rappellerons du moins les principes généraux, ce rappel nous paraissant utile pour expliquer l'objet de notre étude.

Suivant une répartition elassique, on peut distinguer deux groupes de méthodes pour le dosage des matières grasses dans le lait:

$1^{\circ}$ les méthodes volumétriques,

$2^{\circ}$ les méthodes pondérales.

Les méthodes volumétriques consistent à provoquer la sépara-

(1) Paru dans le Volume jubilaire du Professeur Ch. Porcher. 